\title{
A hybrid fiber optic sensing system for simultaneous strain and temperature measurement and its applications
}

\author{
Ginu Rajan, ${ }^{1 *}$ Karolina Mileńko, ${ }^{2}$ Piotr Lesiak, ${ }^{2}$ Yuliya Semenova, ${ }^{1}$ Anna Boczkowska, ${ }^{3}$ Manjusha Ramakrishnan, \\ Kazimierz Jędrzejewski, ${ }^{4}$ Andrzej Domański, ${ }^{2}$ Tomasz Woliński, ${ }^{2}$ and Gerald Farrell ${ }^{1}$ \\ ${ }^{1}$ Photonics Research Centre, Dublin Institute of Technology, Dublin, Ireland \\ ${ }^{2}$ Faculty of Physics, Warsaw University of Technology, Warszawa, Poland \\ ${ }^{3}$ Faculty of Materials Science and Engineering, Warsaw University of Technology, Warszawa, Poland \\ ${ }^{4}$ Faculty of Electronics and Information Technology, Warsaw University of Technology, Warszawa, Poland
}

Received March 24, 2010; accepted March 30, 2010; published March 31, 2010

\begin{abstract}
A hybrid fiber optic sensor by integrating polarimetric fiber sensors and fiber Bragg grating sensors for simultaneous strain and temperature measurement is presented in this paper. Two types of polarimetric fiber sensors are used; a Panda fiber and a polarization maintaining photonic crystal fiber. The polarimetric strain sensors give the average strain and temperature information, while the fiber Bragg grating sensors give localized strain information. Two applications of the proposed configuration are also demonstrated in this paper. In the composite materials three fiber sensors are used while for other engineering applications a temperature insensitive polarimetric fiber sensor can be used together with an FBG sensor to measure strain and temperature simultaneously.
\end{abstract}

Fiber Bragg gratings (FBGs) have enormous potential for strain and temperature sensing in a large range of applications [1]. However, one of the most significant limitations of FBG strain sensors is their high intrinsic temperature cross-sensitivity and their inability to measure strain and temperature simultaneously. Highly birefringent (HB) polarization-maintaining (PM) fiber based fiber-optic sensors are a new generation of sensors known as polarimetric fiber sensors which utilize polarization (phase) modulation within fibers to sense external perturbations [2]. HB polarimetric sensors can be made temperature insensitive but to measure strain they require a means of setting a zero strain reference. To overcome the limitations of a single FBG sensor many schemes for simultaneous measurement of strain and temperature are reported which are based on either superimposed FBGs, twisted FBGs or long period gratings (LPG) [3, 4]. Hybrid approaches by combining FBGs and LPGs [5] and also an FBG together with an extrinsic Fabry-Perot interferometer sensor [6] have also been reported.

In this letter we propose a new method for simultaneous average and localized strain and temperature measurement by using a hybrid approach which involves FBG sensors and highly birefringent polarimetric fiber sensors. We present a hybrid fiber sensing system by combining three types of fiber sensors; a high-

\footnotetext{
*E-mail: ginu.rajan@dit.ie
}

birefringence Panda fiber, a polarization maintaining photonic crystal fiber (PM-PCF) sensor and FBG sensors. The disadvantage of each sensor type can be overcome by using the sensors in a complementary manner to measure temperature independent average strain, temperature and localized strain. A hybrid sensing system for point measurements working in the intensity domain is also demonstrated in the paper, which can be used for a wide range of engineering applications.

The PM fibers used in the system are Panda fiber (FLPM-PF15-04) and PM-PCF (PM 1550-01) both utilized in a polarimetric sensing configuration. The schematic of a general hybrid sensing configuration is shown in Fig 1. A symmetric deformation effect in a single-mode fiber influences the propagation constant $(\beta)$ of every mode because of the changes in fiber length (L) and the effect of stress on the refractive indices of the core and the cladding, which leads to changes in the phase difference between both polarizations of the fundamental mode $\mathrm{LP}_{01}$ [2]. In the case of a Panda fiber, both strain and temperature changes lead to a phase difference between the polarizations of the fundamental mode, while in the case of a PM-PCF the phase difference originates only from longitudinal strain and is insensitive to temperature [7]. Thus by comparing the outputs of polarimetric sensors, average strain and temperature can be obtained simultaneously. In many applications it is important to measure localized strain and temperature. This can be facilitated by using multiple fiber Bragg grating sensors. However, a FBG gives combined strain and temperature information as the wavelength shift in a FBG is sensitive to both strain and temperature. But the influence of temperature can be eliminated by using the temperature information obtained from polarimetric sensors, assuming the temperature is the same across the sample. The average strain in the sample can also be measured by taking the average of localized strain and by eliminating the influence of temperature using the information provided by polarimetric sensors. Finally, the proposed hybrid sensor system has the advantage of increased 
reliability as strain and temperature can be obtained even in the event of failure of one of the sensor types.

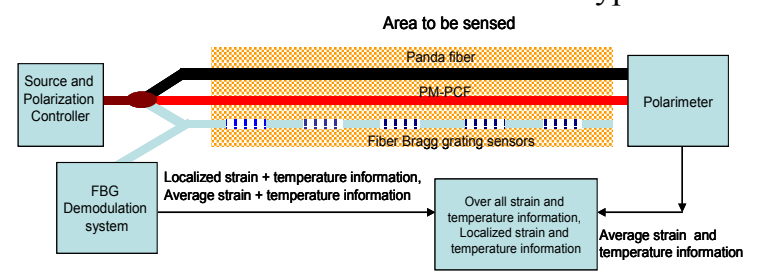

Fig. 1. Schematic of a hybrid sensor sensing using polarimetric and fiber Bragg grating sensors

One of the main applications of the proposed configuration is to monitor strain and temperature inside composite materials. To check the feasibility of implementing such a configuration in composite materials and for a preliminary analysis, a sample has been prepared with all the three types of fiber sensors embedded inside the composite structure. The fabricated composite material sample was $210 \mathrm{~mm}$ long, $25 \mathrm{~mm}$ wide and $3.8 \mathrm{~mm}$ thick. The fabrication process of the composite material sample is shown in Fig. 2. All three types of fiber sensors are embedded in the same layer of the composite material. It is assumed that embedding optical fibers does not modify the material properties of composite structures. However, more research in this direction is currently underway.

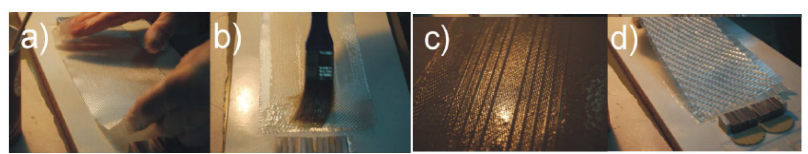

Fig. 2. Sample preparation process: (a) first $2 D$ fabrics layer; (b) epoxy resin; (c) fiber optic sensors layer; (d) next 2D fabrics layer.

The strain sensitivity and phase change of the polarimetric sensors are measured using a PAT9000B polarimeter. A laser source with a wavelength of $1550 \mathrm{~nm}$ is used as the input source. To introduce strain in the composite material sample, the sample is bent by applying force in the middle region of the sample as shown in Fig. 3. The average strain experienced by the sample is calculated analytically [8]. The measured phase change for both the Panda fiber and PM-PCF is shown in Fig. 4. It can be seen that the strain sensitivity of the PMPCF is lower than that of the Panda fiber. However, due to the temperature insensitive nature of the PM-PCF, by comparing its results with that of the Panda fiber, temperature information can also be obtained, if the system is properly calibrated.

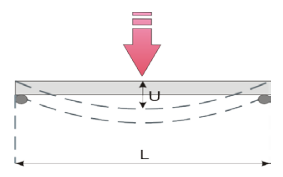

Fig. 3. Schematic of the setup to apply strain to the composite material.

http://www.photonics.pl/PLP

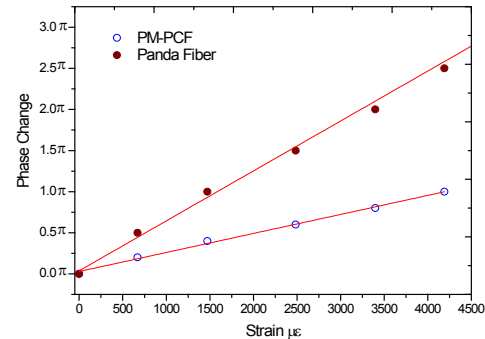

Fig.4. Phase change for polarimetric fiber sensors with applied strain.

To obtain localized strain information, multiple FBGs should be used. In the preliminary analysis we have presented only the data from one of the FBGs with a length of $8 \mathrm{~mm}$ and peak reflected wavelength of 1550 $\mathrm{nm}$, which was placed in the middle of the sample. The wavelength shift due to the applied strain is measured using an optical spectrum analyzer and is shown in Fig. 5.

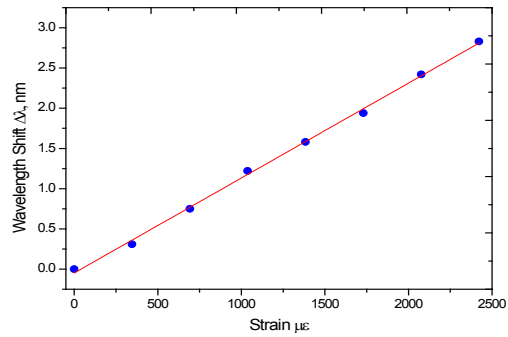

Fig. 5. Measured FBG wavelength shift with applied strain.

The measured wavelength shift includes the influence of ambient temperature which can be eliminated by obtaining temperature information from polarimetric sensors. From the results shown here, we have demonstrated the feasibility of using a hybrid sensing approach for reliable strain measurement unaffected by temperature.

To further underpin the concept of a hybrid sensing system, a point sensor for engineering applications is also demonstrated. In this case the hybrid sensor operates in the intensity domain by converting the polarization and the wavelength information from a photonic crystal fiber sensor and an FBG sensor, respectively, into an intensity variation. The schematic of the configuration is shown in Fig. 6.

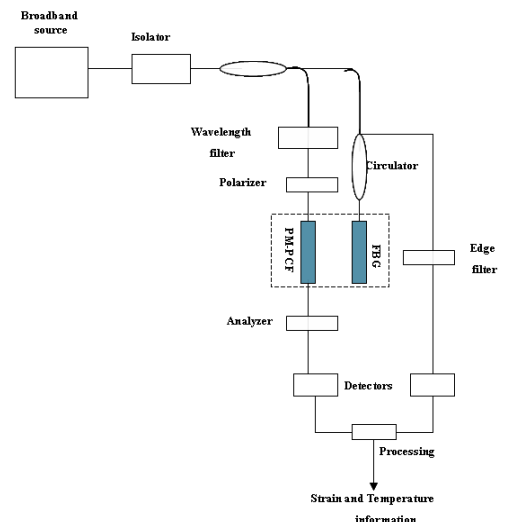

Fig. 6. Schematic of a hybrid sensing system in the intensity domain.

(C) 2009 Photonics Society of Poland 
The PM-PCF used was a buffer stripped PM-1550-01 which was $13 \mathrm{~cm}$ long. The polarized output of the PMPCF was observed at a wavelength of $1550 \mathrm{~nm}$ with the use of a wavelength drop filter. The ends of the PM-PCF were spliced to a standard single mode fiber (SMF28) by using a conventional fusion splicer and the splice loss for both ends was approximately $4 \mathrm{~dB}$. A polarizer and an analyzer at the input and output were used to obtain the strain and temperature induced change in the state of polarization. The peak wavelength of the FBG used was $1560 \mathrm{~nm}$. A fiber circulator was used to direct the reflected signal from the FBG to the edge filter. The edge filter used to convert the strain and temperature induced wavelength variation from the FBG into intensity changes was a macro-bend fiber based edge filter [9]. Two photodiodes were used to measure the intensity variation.

The strain response slope of the intensity spectra of both the FBG sensor and the polarimetric sensor are equalized by adjusting the slope of the edge filter. An edge filter with a slope of $0.25 \mathrm{~dB} / \mathrm{nm}$ converts the wavelength information from the FBG sensor, whose peak wavelength is at $1560 \mathrm{~nm}$ and produces the same intensity variation as that of the polarimetric sensor at $1550 \mathrm{~nm}$ at constant temperature $\left(20{ }^{0} \mathrm{C}\right)$. Fig. 7 shows the intensity variation for both sensors when step changes in strain are applied. The strain step of $192 \mu \varepsilon$ was limited by the resolution of the translation stage used to apply strain. From the figure it is clear that for both sensor systems the intensity variation is linear and also has the same magnitude.

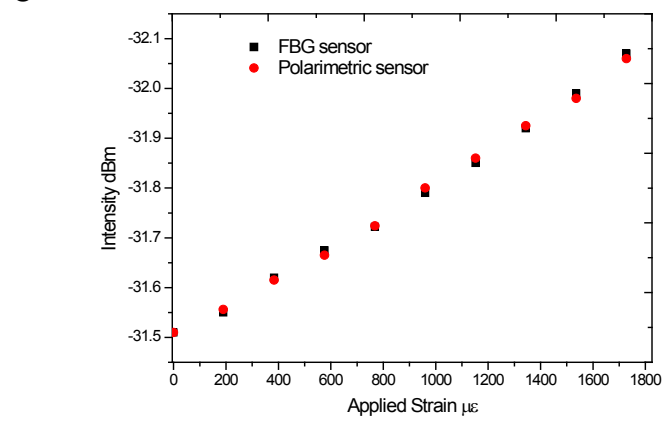

Fig.7. Strain response of both polarimetric and FBG sensor in the hybrid configuration.

To measure the temperature response, the sensors are attached to a Peltier cooler which is controlled by a temperature controller. The temperature of the sensors is varied from $20{ }^{0} \mathrm{C}$ to $70{ }^{0} \mathrm{C}$ at intervals of $5{ }^{\circ} \mathrm{C}$ and the intensity outputs of both sensors are monitored. The results are shown in Fig. 8. From the figure it is clear that the PM-PCF based polarimetric sensor is insensitive to temperature. Thus by configuring both sensor systems together as a hybrid sensor both strain and temperature can be measured simultaneously. The PM-PCF polarimetric sensor provides strain information and from the difference between the change in the output intensity of the PM-PCF sensor and the FBG sensor, temperature information can be obtained.

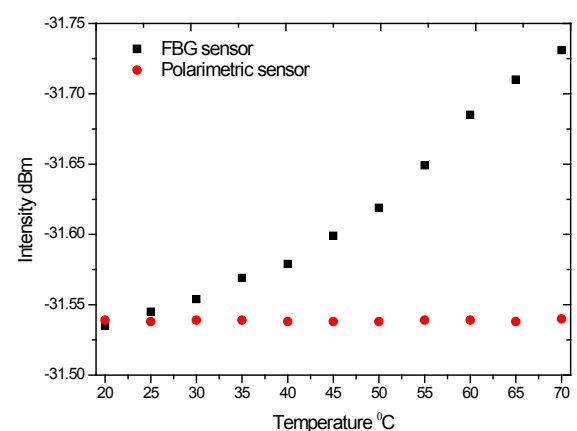

Fig.8. Temperature response of both polarimetric and FBG sensor in the hybrid configuration.

In this letter a hybrid sensor is proposed and demonstrated by combining polarimetric sensors and FBG sensors. Two types of polarimetric fiber sensors are used; a strain and temperature sensitive Panda fiber and a temperature insensitive PM-PCF to measure the average temperature independent strain. For localized strain measurements, FBGs can be utilized and temperature influence can be eliminated by comparing the results with those of the polarimetric fiber sensors. Two sample applications of the hybrid sensing systems are also demonstrated; one for structural monitoring in composite materials where three fiber types are used and the other as a point sensor for engineering applications where localized strain information is required by utilising two fiber sensors and an intensity domain interrogation system. The proposed hybrid configuration can be effectively used to measure strain and temperature more reliably and can be implemented in a wide range of sensing applications.

This work was supported by the Polish Ministry of Science and Higher Education through The National Centre for Research and Development and by Enterprise Ireland under the grant ERA-NET MATERA.

\section{References}

[1] K. O . Hill, G. Meltz, J. Lightwave Technol. 15, 8 (1997)

[2] T. R. Wolinski, P. Lesiak, A. W. Domanski, Bull. Pol. Ac. Tech 56, 2 (2008).

[3] O. Frazao, L. A. Ferreira, F. M. Araujo, J. L. Santos, J. Opt. A: Pure Appl. Opt 7, (2009).

[4] K. B. Samer, T. Sub, K. T. V. Grattan, Sensors and Actuators 144, (2008)

[5] H. J. Patrick, G. M. Williams, A. D. Kersey, J. R. Pedrazzani, IEEE Photon. Technol. Lett 8, (1996).

[6] R. De Oliveria, C. A. Ramos, A. T. Marques, Computers and Structures 86, (2008)

[7] D. H. Kim, J. U. Kang, Opt. Engg 46, 7(2007).

[8] A. W. Domanski etal, Acta Physica Polonica A 116, 3(2009).

[9] Q. Wang, G. Farrell, T. Freir, G. Rajan, P. Wang, Opt. Lett 31, (2006). 